\title{
Challenges and Support when Introducing AM in HPDC Tooling
}

\author{
Roland STOLT ${ }^{\mathrm{a}, 1}$, Fredrik ELGH ${ }^{\mathrm{a}}$ and Anders JARFORS ${ }^{\mathrm{b}}$ \\ andustrial Product Development, Production and Design, Jönköping University, \\ Sweden \\ ${ }^{\mathrm{b}}$ Materials and Manufacturing, Jönköping University, Sweden
}

\begin{abstract}
When manufacturing tooling inserts for HPDC (High Pressure Die Casting), several manufacturing steps such as milling, heat treatment, electro discharge machining and finally surface treatment are involved. By instead manufacturing the insert by SLM (Selective laser melting), the process is expected to be quicker and with less material waste compared to the traditional manufacturing. Examples of other expected advantages is higher product variant flexibility and the possibility of making conform cooling channels, extending the die life. However, the insert is part of a die system involving many components. The insert cannot be designed and manufactured without considering the complete die system. This paper seeks how to integrate the insert design in the die assembly design. This is done via an example component and in cooperation with die manufacturing firms. The result is that the printing is a minor step of the total manufacturing process and that special design considerations needs to be taken for an SLM insert. New die concepts are needed that will minimise the amount of material, reduce the tolerance and surface demand and support the subdivision of the die into several printed parts.
\end{abstract}

Keywords. SLM, Additive Manufacturing, Design, Tooling, Modular

\section{Introduction}

Dies for high pressure die casting, HPDC consist of many different components and are often designed and manufactured by specialised die manufacturers. An important component in the die assembly is the die insert in which the molten metal is injected. To cope with the high stresses, they are made from hardened high-grade tool steels. The design and manufacturing of these highly advanced components includes many steps, making them time consuming and resource demanding to manufacture. With the advent of additive manufacturing AM printing techniques such as selective laser melting SLM, suggestions have been made to instead print the die inserts. Several advantages are then expected such as use of less material, the possibility to print conformal tooling channels, reducing the manufacturing time and increasing the flexibility to product variants [1]. However, it is not clear how the design and manufacture of printed dies will compare to the traditional way of design and manufacture of die assemblies. What are the main differences and what pros and cons that can be expected when printing the die inserts? What support is needed when designing for SLM printing? In this paper, an investigation is made to answer these questions. It involves an example where an existing HPDC die

\footnotetext{
${ }^{1}$ Corresponding Author, Email: roland.stolt@ju.se.
} 
assembly for manufacture of fatigue test samples is redesigned for manufacture by the SLM process. A supplier of conventional HPDC tooling has provided data allowing a comparison between printed and conventional die assemblies.

\section{Literature}

$\mathrm{AM}$ is a relatively resent manufacturing method and the research community has currently a very high interest in it with a multitude of publications emerging. The publications cover all aspects of AM from the design of components, development of materials to simulating the process and assuring the quality of the manufactured parts. In [2] an overview is given, accounting for the state of art in AM. This review is broad and includes most AM materials and processes. In this current paper, the SLM process is central. It is a bed fusion process. In [3] the design process of SLM parts is elaborated. It includes several design activities that are typical for SLM components. These are aimed at both reducing the cost and increasing the performance of the components. It includes keeping the material volume and build height low (minimize print time and weight) and to determine the best part orientation minimizing the amount of support material needed. They apply the method to an example aerospace component, significantly reducing its weight. An application of a design method taking the same considerations into account is made by [4]. They show how a hydraulic manifold is designed taking weight minimisation and the orientation of the part in the printer into account. Minimising the amount of material in SLM parts is very important both from a manufacturability perspective, reducing the print time and risk of deformation and from a product performance perspective where light weight is key in many applications. In the two referenced papers, topology optimisation TO is central in the design process. TO is a computational method aimed at distributing the stresses in the part by altering its topology so that the least amount of material needed for carrying the load is used. Examples of the application of TO to SLM parts with the objective of minimising their weight is plentiful $[5,6]$. Either material that is subjected to low stress is removed completely, or it is replaced with lattice structures. Lattice structures are structures for which the density can be controlled [7].

When designing die inserts, the design strategies from above apply. However, the weight of the die insert is not as crucial from a functional perspective as for example in aerospace applications. The amount of material is primarily reduced to keep the printing time low and to reduce the risk of part distortion. From an energy consumption perspective however, it is beneficial to reduce the mass. Energy is saved when operating the die in production. This is shown by [8] who propose a light weight die design based on trusses. It is a general lightweight design, not directly intended for printing. They verify by FEA calculations that their truss design can withstand the stresses and they also calculate the thermal balance, including spraying of the die. Their truss design is compared to a conventional die. They found that the lightweight die is expected to save several megajoules in production because of the smaller thermal mass. They also found that the lightweight die is expected to be slightly less capable of keeping the wall thickness tolerances of the produced components. In reference [9] two versions of a die of the same component have been designed and compared. One is a conventional die and the other is a lightweight die for manufacture by SLM. They find that it is possible to design a SLM version keeping the stresses within the permissible range. The stresses are highest when the filling pressure is at its peak. 
In [10] the effect of conformal cooling channels in HPDC dies has been studied. They conclude that the temperature distribution improves compared to conventional tooling and thereby the surface defects are reduced. In [11] the thermal conductivity of lattice structures has been investigated and the authors have devised a strategy for optimising the conductivity. This is expected to be of high importance in SLM manufactured dies.

To conclude from the literature study, several new considerations will emerge when die insets are designed for the SLM process. It includes strategies for designing conform cooling channels, devising light weight lattice with high thermal conductivity and predicting the stresses. Predicting the expected materials properties and finding the best orientations in the printer is also of high importance.

\section{Designing the HPDC tooling assembly}

In practice, when designing a die assembly, a CAD-model (usually STEP or Parasolid) of the intended component to be produced serves a starting point. In addition, there are plenty of other considerations taken. This is handled in engineering drawings and contractual documents exchanged by the tooling firm and the customer. How this is done in detail has been investigated for three different tooling firms [12]. At one of the firms, employing around 10 people, complete tooling assemblies are designed and manufactured. It is this firm that has been interviewed for a description of their design and manufacturing process. They have also provided data on manufacturing lead times for this paper. They do not have any SLM printer, and lack experience in the process. Their production equipment consists mainly of CNC and EDM machines. The tooling firm uses Siemens NX Tool wizard to support and increase the productivity and precision in their design process. Below is a list of the different steps that are taken after they have received the $\mathrm{CAD}$-model and other specifications from the customer:

1. The parting plane is planned to determine what part of the geometry is formed in the fixed and what is formed in the ejector die. It is checked that tapers are present and that they allow ejection. If needed, moving members operating on different parting planes than the main are planned. These are moved by hydraulics or transverse pins.

2. The projected area of the part determines the required size of the HPDC machine. It must meet the requirement on locking force to counteract the hydrostatic pressure. Usually 80 tons and upwards are used.

3. The sizes of the tool steel slabs from which the inserts will be manufactured can now be determined. These are supplied in standardized sizes. A zone of at least $70 \mathrm{~mm}$ is projected around the perimeter of the part. It is this outline and the depth of the cavity that determines the size of the slab. The closest larger standard size is selected.

4. The insert is to be fitted in a die that is larger than the insert. These dies are sometimes termed bolsters. They are also formed from a standard size tool steel slab of enough size to accommodate the inserts. A good fit (grade 6) is required between insert and bolster.

5. The ingate, runners and cooling channels are designed. The filling and ejection cycles are planned. 
6. The design is verified by simulating the filling of the dies and the thermal balance. Specialized software is used. The shrink compensation is determined.

7. The die assembly needs ejector pins, replaceable cores, alignment elements, moving members and several other components. These are designed and verified. Mould wizard provides standardised components and routines to assist in this process.

8. The manufacturing operations such as milling and EDM operations are planned. CNC code is generated. The die assembly can now be sent for manufacturing.

In order to design the inserts for manufacturing by SLM, additional considerations need to be taken. Steps 1 and 2 will be the same, but step 3, will have to be modified when designing SLM inserts. The steps a-h below outlines the considerations that must be taken when designing an insert for the SLM process.

a) Decide the printed volume by component size + marginal. The printed volume must be accommodated in the print envelope of the intended printer.

b) Design fastening elements between insert and bolster. Add positioning elements.

c) Design conform cooling channels in the insert.

d) Minimise the amount of material in the insert.

e) Determine the printing parameters and orientation in the printer.

f) Check that the stresses in the insert do not exceed the maximum permissible.

g) Design a fitting between bolster and insert. At least class 6 is needed. The fitting must be sufficiently tight to prevent metal ingress between die and insert. The insert top surface the die top surface should be flush. Otherwise the sealing surface between the tooling halves will become too small.

h) Plan the post treatment of the die inserts. This should include support structure removal, heat treatment, machining to tolerance and possibly EDM to obtain a dull finish tool surface.

\subsection{Example}

To try the prescribed a-h steps, a HPDC die assembly for manufacturing of aluminium fatigue test samples has been chosen as an example. The complete assembly is shown in figure 1.

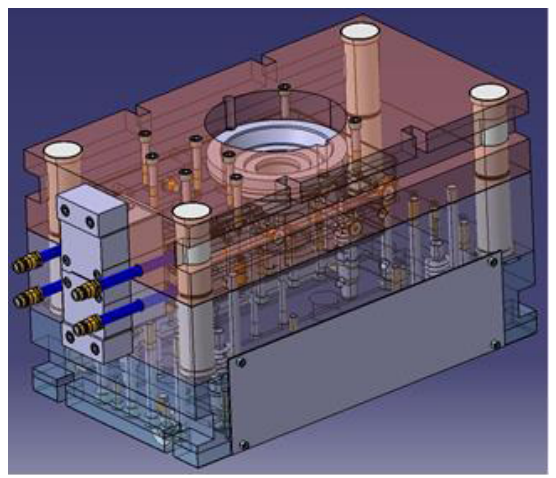

Figure 1. Complete die assembly. (see Acknowledgement) 
The objective is to modify the assembly for manufacture by SLM. Since it is an existing tool, there is no need to go through steps 1-8. Steps a-h will be conducted to convert the insert design to SLM. The existing fixed and ejector die inserts are shown in figure 2 on the left-hand side.

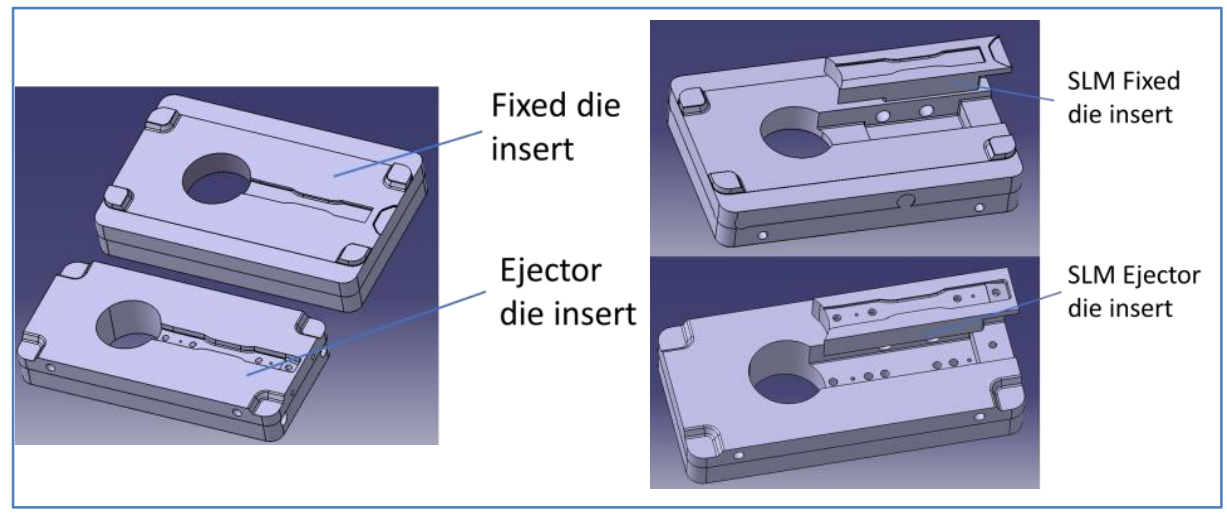

Figure 2. Die inserts in existing tool.

The dimensions of the largest of these inserts is $420 \times 270 \times 70 \mathrm{~mm}$. This is too much for the intended printer, a 3DSystems ProX ${ }^{\circledR}$ DMP 300 which has and envelope of $(250 \mathrm{x}$ $250 \times 330 \mathrm{~mm}$ ). This is solved by making an SLM insert in the existing insert. The test samples are to be manufactured in several different versions with varying thicknesses i.e. $6.3,10.2$ and $20.3 \mathrm{~mm}$. By letting the part of the insert geometry that varies comprise one part (module), the variants can be produced with a minimum variation of the die. A pocket is milled in the existing insert to accommodate the SLM inserts. To create the SLM inserts, an offset of about $20 \mathrm{~mm}$ is made around the form cavity These volumes are as shown in figure 2 on the right-hand side. Creating these volumes means completing step (a).

In the SLM ejector die insert, the ejector pins are arranged as in the original. Holes are added through the SML insert to accommodate the ejector pins. For joining the inserts and SML inserts bolts and threads are added. An extra positioning element is added on the ejector side. On the fixed side the positioning is obtained from the existing geometry. Step (b) is now complete.

\subsection{Design of Cooling channels}

The original inserts had straight drilled cooling channels, $\varnothing 15 \mathrm{~mm}$. These are kept and for the SLM inserts separate cooling circuits $\varnothing 12 \mathrm{~mm}$ are added. The reason for separating the cooling circuits is that it not possible to connect them. According to the company, there is currently no method of sealing the transition. Oil would inevitably leak, creating a fire hazard. Thus, there will be one cooling circuit in the insert and an additional one in the SLM insert with separate oil pumps. The SLM cooling channels are placed so that a minimum of $6 \mathrm{~mm}$ material is left between the surface in contact with molten metal and the cooling channel. It is assumed that $6 \mathrm{~mm}$ material will be enough to keep the oil and the molten metal separate under the stresses that the inserts are subjected to. However, this assumption needs to be verified. The layout of the cooling circuits of the SLM insets are seen in the figure 2. Note that on the ejector side it was necessary to change the shape of the cooling channel section from circular to elliptic to 
accommodate the ejector pins. The cross-sectional area is constant in the cooling channel. Step (c) is now complete.

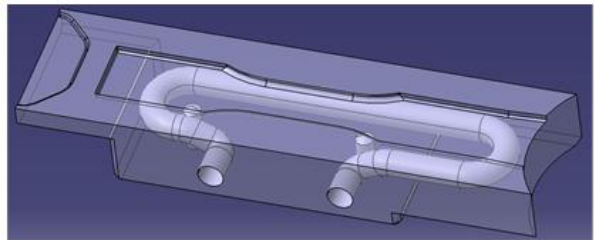

Fixed side

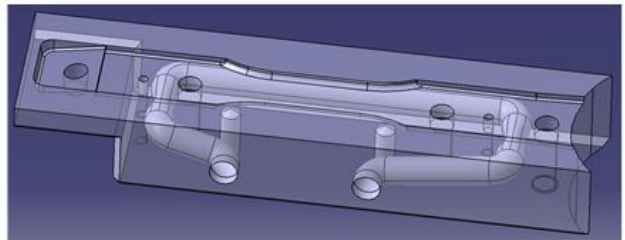

Ejector side

Figure 3. SLM Insets with conform cooling channels.

The material volume of the fixed side insert is $382 \mathrm{~cm}^{\wedge} 3$ and the ejector side insert is $404 \mathrm{~cm}^{\wedge} 3$. The thickest section in the inserts is $35 \times 65 \mathrm{~mm}$. An estimate of the print time is 85 hours on the ProX printer. The printing time is quite long due to a large material volume. The $6 \mathrm{~mm}$ overall material thickness in the die made it impossible to shell either of the parts completely. Instead, voids could be created in the thickest parts of the inserts, bringing down the volume to $359 \mathrm{~cm}^{\wedge} 3$ on the fixed side and $381 \mathrm{~cm}^{\wedge} 3$ on the ejector side (step d). The powder can either remain inside the voids or drain holes can be added to remove it. Note that adding the voids has only slightly reduced the material volume. With a more detailed analysis it is likely possible to further reduce the material volume for example by using topology optimisation and adding lattice infill and yet fulfilling the requirements.

The print preparation made in the software $3 \mathrm{dXpert}$ is shown in figure 4 (step e). With the reduced volume, the expected printing time is now 66 hours. The material in red is the support material. The parts have been tilted to minimise overhang.

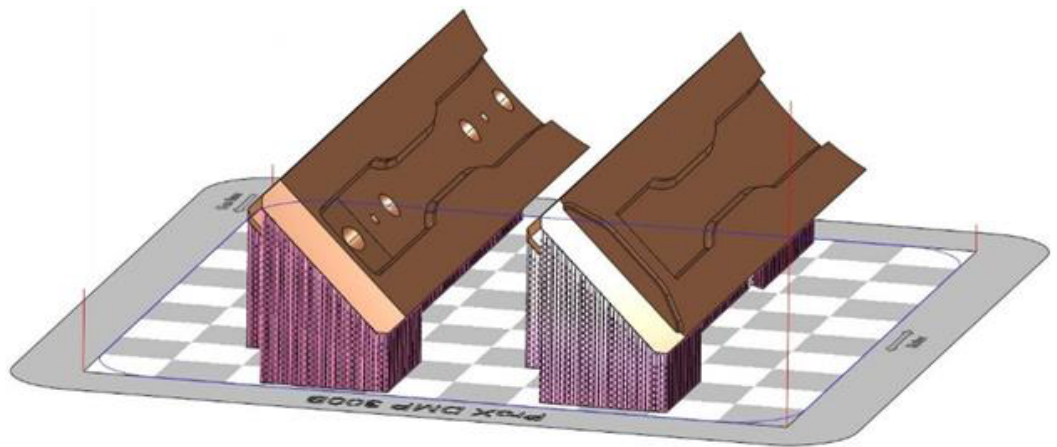

Figure 4. The print preparation in 3DXpert.

\subsection{Assessing the strength of the inserts}

It should be noted that software vendors have started to provide process simulation capability to predict the expected deflections and internal stresses.

To check that the SLM inserts will not break during the production cycles some calculations are made. It is checked using a simple static FEA analysis, that the injection pressure of 800 bars and the closing force will not cause any plasticity in the material.

It is targeted that the inserts will have a working temperature of 270 degrees Celsius. Step (f) is now complete. 


\subsection{Planning the post processing}

There is extensive post processing required on the SLM inserts (step h). The expected dimensional tolerance from the print is about $50 \mu \mathrm{m}$ on the ProX 300 DMP (www.3dsystems.com). This is not enough since a class 6 tolerance on a $30 \mathrm{~mm}$ length is $16 \mu \mathrm{m}$ according to ISO 2768. The expected surface roughness from the print is in the order of $8 \mu \mathrm{m}$. The requirement is a dull finish of 4-6 $\mu \mathrm{m}$. A planning of the machining sequence is needed. This cycle include machining of the surfaces were the support material is located removing the support material in this operation. The surface (Ref A in the figure 5) below will serve as a reference when machining. Step $\mathrm{h}$ is now completed.
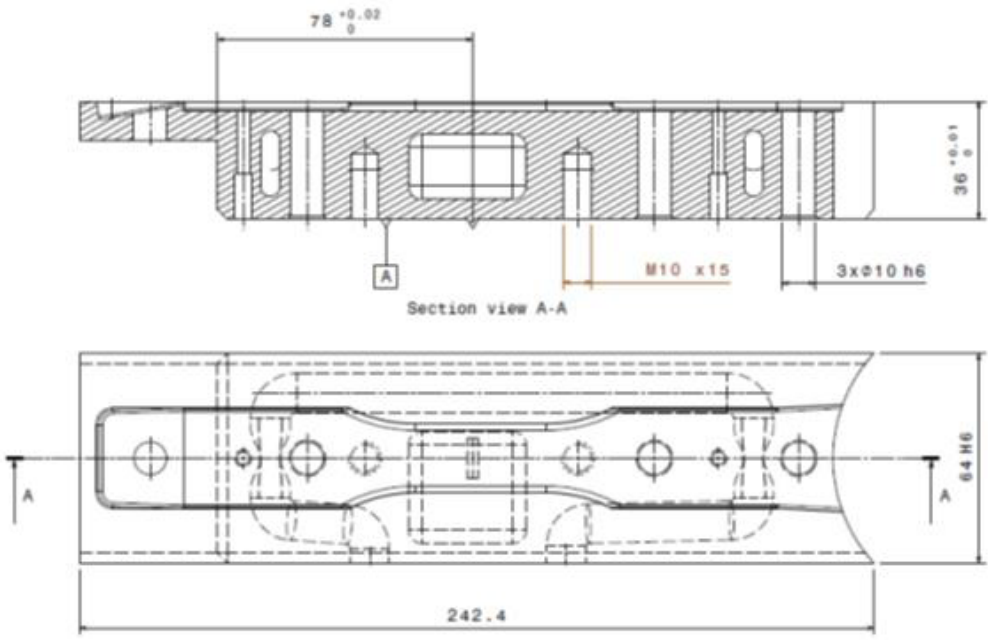

Figure 5. Drawing view of ejector SLM insert.

\subsection{Lead times in manufacturing of the die assembly}

The CEO and head of production of the tooling company were interviewed on what time is usually spent on the different manufacturing operations. It turned out that the most time-consuming part is indeed the insert. The part must be hardened and the requirements on tolerances is higher than any of the other components in the assembly. For a standard HPDC part die system, about 8 weeks is spent on the insert. The first rough machining of the non-hardened material is quick, around 20hours. This is in fact less than the expected printing time. The hardening of the tool takes one week at a supplier. The following fine machining of the hardened material, the EDM and the final polishing of the surface takes the better part of the 8 weeks. The polishing of the tool inserts represents around one week of manual work. The bolsters are less time consuming. The company allot 40 to 80 hours for their manufacturing. In all, it is the lead time of the insert that is decisive for the total time spent on the die assembly.

\section{Discussion}

In the introduction of this paper it was asked what support is needed when designing HPDC dies for SLM printing and what the main differences are between printing and 
conventional manufacturing of die inserts. The answer to the support question is that since it is well known that the material volume of printed die components must be kept low to keep the printing time low, designers need to know how the material thickness can be reduced without compromising the safety and die life. In the example presented in the paper it was assumed (but not verified) that $6 \mathrm{~mm}$ is an enough shell thickness. The geometry of the inserts then became mostly thick although it was possible to accommodate some internal voids to reduce the material volume. The geometry of the current inserts is not ideal from a printing perspective. Designers will need recommendations on how to design the underlying support structure to have sufficient thermal conductivity and strength for a minimum material volume.

Further, designers need support in how to attach the inserts in the die assembly. It seems that both dimensional tolerances and surface conditions of printed parts currently are too poor to make the needed press fit. This is the reason why much post-treatment is needed. There are perhaps alternative ways of designing so that the requirements on the fitting would be less demanding.

The interview with the tooling company indicated that the die insert is the most timeconsuming component of the die assembly to manufacture. Consequently, it is decisive for the total lead time. Finding ways of reducing the number of manufacturing steps will therefore have a direct impact on the total lead time. Consequently, reducing the amount of post-treatment on the printed inserts is important.

The assumed $6 \mathrm{~mm}$ wall thickness resulted in an estimated printing time of 66 hours. It is uncertain if this can compete in terms of lead-time with traditional manufacturing especially since there will be many post treatment steps needed. The major motivation for printing will then be the possibility to make conformal cooling channels in the insert. It remains to examine the effect of this on the die performance. A reasonable assumption is that it will be possible to keep an even die temperature which will reduce the thermal stresses in the die adding to the expected die-life.

It should be noted that it would not have been possible to accommodate straight drilled channels in the current insert volumes. If the inserts were to be manufactured conventionally, they would have to be made larger than the printed version.

In the example the size of the inserts is near the maximum capacity of the printer. This can be expected to be a problem when printing die inserts. The envelope of CNC machines is currently much bigger than that of SLM printers. On way forward is perhaps subdividing the inserts into several printed parts. However, there are several challenges in this. The parts need to have good fittings between them preventing metal ingress, and they will need separate cooling circuits since there is no feasible way of making a reliable seal between them.

\section{Conclusions and future work}

It can be concluded that support is needed for designers to reduce the material volume in die inserts without compromising the safety and die life. Both new die concepts and assessment methods needs to be developed. Currently, the primary motivation for SLM printing is the possibility to make conformal tooling since there is too much post treatment needed on printed parts to compete on lead-time. Efforts should be put into reducing the amount of post-processing to bring down the lead time for manufacturing the inserts which is the bottleneck in die assembly. 
It can also be concluded that no feasible concepts for sealing transitions between cooling channels sub-parts are available. Therefor separate cooling circuits are needed adding to the complexity of the tooling assembly.

The inserts had not been printed at the time that this conference paper was due. It remains to print them and measure the actual dimensional outcome to determine how much post treatment that will be needed. It will for example perhaps be possible to finalise the inserts without any EDM of the tool surface since the expected surface roughness from SLM only is expected to be a little coarser the required. Testing them in actual HPDC will revile if there is a tendency for the metal to stick to the slightly coarser surface.

Work should also be devoted to developing new concepts for die insert design with a low material volume and reduced requirements on tolerances. A net-shape printed die should be envisioned.

\section{Acknowledgements}

Participating companies and the Swedish Knowledge foundation are greatly acknowledged for financing and contributing to this research under the project "Distinct". A special thanks is directed to Olof Granath in Jönköping University who did the original design of the HPDC tooling and shared valuable knowledge on die design.

\section{References}

[1] Y. Queudeville, et al., Decision and design methodologies for the lay-out of modular dies for highpressure-die-cast-processes. Materials Science Forum, 2009. 618 619: p. 345-348.

[2] M.K. Thompson et al., Design for Additive Manufacturing: Trends, opportunities, considerations, and constraints. CIRP Annals - Manufacturing Technology, 2016. 65(2): pp. 737-760.

[3] S. Hällgren, L. Pejryd and J. Ekengren, (Re)Design for Additive Manufacturing. Procedia CIRP, 2016. 50(Supplement C): pp. 246-251.

[4] J. Schmelzle et al., (Re)Designing for Part Consolidation: Understanding the Challenges of Metal Additive Manufacturing. Journal of Mechanical Design, Transactions of the ASME, 2015. 137(11), No. 111404.

[5] Ranjan, R., R. Samant, and S. Anand, Integration of Design for Manufacturing Methods with Topology Optimization in Additive Manufacturing. Journal of Manufacturing Science and Engineering, Transactions of the ASME, 2017. 139(6), No. 061007.

[6] S.N. Reddy, et al. Application of topology optimization and design for additive manufacturing guidelines on an automotive component. in Proceedings of the ASME Design Engineering Technical Conference. 2016, https://doi.org/10.1115/DETC2016-59719.

[7] A.H. Azman, Method for integration of lattice structures in design for additive manufacturing. $\mathrm{PhD}$ thesis, Université Grenoble Alpes, 2017.

[8] S. Müller et al., An Initial Study of a Lightweight Die Casting Die Using a Modular Design Approach. International Journal of Metalcasting, 2018. 12(4): pp. 870-883.

[9] M. Baradaran and A.T. Pradeep, HPDC Die design for Additive Manufacturing, in Product Development. 2018, Jönköping University.

[10] A. Armillotta, R. Baraggi, and S. Fasoli, SLM tooling for die casting with conformal cooling channels. International Journal of Advanced Manufacturing Technology, 2014. 71(1-4): pp. 573583.

[11] L. Cheng, et al., Coupling lattice structure topology optimization with design-dependent feature evolution for additive manufactured heat conduction design. Computer Methods in Applied Mechanics and Engineering, 2018. 332: pp. 408-439.

[12] R. Stolt, S. André, and F. Elgh, Introducing Inserts for Die Casting Manufactured by Selective Laser Sintering, in 28th International Conference on Flexible Automation and Intelligent Manufacturing, 
FAIM 2018, Columbus, United States, 11 - 14 June 2018, G.S.F.F.C. Dušan Šormaz, Editor. 2018, Elsevier. p. 309-316. 